\title{
TOKAMAK CONFINEMENT PROJECTIONS AND PERFORMANCE GOALS*
}

\author{
N. A. UCKAN \\ Oak Ridge National Laboratory \\ P.O. Box 2009 \\ Oak Ridge, Tennessee 37831-807?
}

(615) $574 \cdot 1354$

\section{ABSTRACT}

One key quantity to be determined in the design of burningplasma devices (CIT, ITER, reactors, etc.) is the level of plasma current $(I)$ required to meet the desired plasma performance goals (ignition, high $Q$, etc.) and device objectives (fusion power, wall loading, current drive power, etc.). It is shown that these goals and objectives can be expressed in terms of the "figure-of-merit" parameter $I A^{\alpha} / R^{x}\left[\sim f\left(L B^{y}\right)\right]$, where $A$ is the aspect ratio, $R$ is the major radius, $L(=R, a)$ is the characteristic length, $B$ is the toroidal magnetic field on axis, and the exponents $\alpha \sim 1 \pm 0.5$ and $x \sim 0-0.5(y \sim i-2)$ depend on the confinement assumptions and operational limits. To reach ignition or high $Q$, the main goal is to optimize $I A^{\alpha} / R^{\mathrm{x}}$, subject to other engineering design constraints. In a CITlike device (with $R-2 \mathrm{~m}, \mathrm{\kappa}-2, q_{\psi} \geq 3$ ), the ignition requirement is $l(A / 3)^{\alpha} \sim 9-15$ MA for "enhanced" L-mode (H-mode) confinement scaling expressions; an ITER-like device (with $R \sim 5-6 \mathrm{~m}, \mathrm{k} \sim 2, q_{\Psi} \geq 3$ ) would require $I(A / 3)^{\alpha}-15-25 \mathrm{MA}$. These requirements are embodied in the present $\mathrm{CIT}$ (with $I \sim 11 \mathrm{MA}, A \sim 3.25$ ) and ITER (with $I-18-22, A \sim 3.1-2.6$ ) designs.

\section{INTRODUCTION}

Energy confinement is a major issue for the nextgeneration, buming-plasma devices [Compact Ignition Tokamak' (CIT), International Theninonuclear Experimental Reactor $^{2}$ (ITER), etc.]. The physics of energy transport in tokamaks is not yet fully understood. Therefore, the energy confinement time in these buming-plasma devices has to be estimated from extrapolations of the available experimental data base by using empirically developed scaling expressions ${ }^{3}$ as well as scalings derived from various theoretical models ${ }^{4}$ for the dominant transport mechanisms.

* Research sponsored by the Office of Fusion Energy. U.S. Department of Energy, under contract DE-ACO5$840 R 21400$ with Martin Marietta Energy Systems, Inc.
The selection of a particular scaling or transport model has a large impact on the design and parameter choices. Thus, the identification and formulation of "figure-of-merit" parameters such as $T n \tau_{E} \propto f\left(I A \alpha / R^{x}\right) \propto f\left(L B^{y}\right)$ are useful in guiding the design efforts to optimize energy confinement and $1 v$ establish trade-offs between plasma size $(L=a$, $R$ ), current $(I)$, field $(B)$, aspect ratio $(A)$, etc. In this paper we formulate these figure-of-merit parameters and assess the confinement capabilities of CIT and ITER.

Unless otherwise stated, all units are mks, with $T$ in $\mathrm{keV}, I$ in $\mathrm{MA}$, and power in MW.

\section{PERFORMANCE GOALS AND FIGURES OF MERIT}

There are several performance indicators for tokamaks; the level of plasma current $(\Lambda$ is one of the most important because of the favorable scalir ; of confinement time $\tau_{E}$ and limits on plasma beta $\beta_{\text {crit }}$ and density $\langle n\rangle_{\max }$ with increasing $I$. One key quantity to be determined in the design of CIT and ITER (or any buming-plasma devices) is the rlevel of plasma current equired to meet the desired plasma performance goals $\left(T n \tau_{\mathrm{E}}-\right.$ ignition, high $Q$, etc.) and machine objectives (fusion power $P_{\text {fus }}$, wall loading $\Gamma_{\mathrm{w}}$, current drive power $P_{\mathrm{CD}}$, etc.).

Here we define a fusion-related parameter

$$
T_{10} n_{20} \tau_{\mathrm{E}}=\langle T / 10 \mathrm{keV}\rangle\left\langle n_{d} / 1020 \mathrm{~m}^{-3}\right\rangle \tau_{\mathrm{E}}
$$

where $\left\langle n_{e}\right\rangle$ is the volume-averaged electron density anc: $<T>$ is the density-weighted average temperature $\left(T_{\mathrm{i}} \approx T_{\mathrm{e}}\right.$ $\approx T$ ). The importance of this parameter derives from the fact that, in the temperature range $7<T<20 \mathrm{keV}$, the D-T fusion reaction rate coefficient $\langle\sigma v\rangle \propto T^{2}$, and a measure of confinement capability $M \propto P_{\alpha} / P_{\text {loss }} \propto T_{10} n_{20} \tau_{\mathrm{E}}$, where $P_{\alpha}$ is the total alpha power and $P_{\text {loss }}=P_{\text {cond }}+$ $P_{\text {rad }}$ is the power lost by conduction and radiation. For

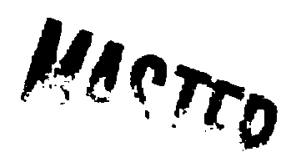


relatively broad (square-root parabolic density and parabou iemperature) profiles with $Z_{\text {cff }} \sim 1.5$ (5\% thermal alphas filus carbon and oxygen impurities), the ignition requirement ${ }^{5}$ is $T_{10} n_{20} \tau_{E}-3 \pm 0.3$ for $\langle T\rangle-7-20 \mathrm{keV}$. With a Murakami-Hugill $\left(n_{\mathrm{mu}}-1.5 B / R q_{*}\right)$ or Greenwald $\left(n_{\mathrm{gr}}\right.$ $0.27 / / a^{2}$ ) type limit for density, the Troyon limit for beta $\left[\beta_{\text {crit }} \sim C l / a B, C \sim(2.5-3) \%\right]$, and $q_{\psi}>3-3.5$ for MHD stability, the optimum temperature for ignition, is typically $-10 \pm 2 \mathrm{keV}$ for many of the confinement scaling models. ${ }^{3-11}$

For a wide range of confinement scaling models of the form $\tau_{\mathrm{EOH}}$ (neo-Alcator) $\propto n L^{3}$ and/or $\tau_{\mathrm{Eaux}}(\mathrm{L}-/ \mathrm{H}$-mode $) \propto$ $L I f(P)$ with $f(P)-P-\gamma$ or $\sim\left(C_{1}+C_{2} / P\right), T n \tau_{\mathrm{E}} \propto$ $f\left(/ A^{\alpha} / R^{\mathrm{x}}\right)$. An example of the power law form of $\tau_{\mathrm{E}}$ is the Goldston scaling ${ }^{6}$

$$
\begin{aligned}
& \tau_{\mathrm{E}}=\tau_{\mathrm{G}}=0.037 / P^{-0.5} R^{1.75} a^{-0.37} \kappa^{0.5}\left(A_{\mathrm{i}} / 1.5\right)^{0.5} \\
& T_{10^{n_{20}} \tau_{\mathrm{E}}=2.53 \times 10^{-4}\left(I A^{1.37} / R^{0.12}\right)^{2}}
\end{aligned}
$$

where $A_{\mathrm{i}} \approx 2.5$ is the average atomic mass and $P=W / \tau_{\mathrm{E}}$ $=P_{\mathrm{aux}}+P_{\mathrm{OH}}+P_{\mathrm{a}}-P_{\mathrm{rad}}=9 n_{20} T_{10} R a^{2} \mathrm{\kappa} / \tau_{\mathrm{E}}$ is the net heating power. An example of the offset linear form is the Rebut-Lallia scaling ${ }^{10}$

$$
\begin{aligned}
& \tau_{\mathrm{E}}=\tau_{\mathrm{RL}}=C_{\mathrm{R}} / l^{1.5}+C_{\mathrm{L}} n_{20} 0.75 I^{0.5} B^{0.5} l^{2.75} / P \\
& \tau_{10^{n_{20}} \tau_{\mathrm{E}}} \propto I^{3} A^{2} / R^{0.5} \propto\left(I A^{0.67} / R^{0.17}\right)^{3}
\end{aligned}
$$

where $l=\left(R a^{2} \kappa\right)^{1 / 3}, C_{\mathrm{R}}=\left(0.02 .4 / Z_{\text {eif }} 0.5\right)\left(A_{\mathrm{i}} / 2\right)^{0.5}$, $C_{\mathrm{L}}=0.29 Z_{\mathrm{eff}} 0.25\left(A_{\mathrm{i}} / 2\right)^{0.5}$, and $T_{n} \tau_{\mathrm{E}}$ is evaluated at the betu limit $(\propto / / a B)$.

Table I lists [for a given plasma shape $(\kappa, \delta)$ and $q_{\psi}$ ] the ignition/burn-related plasma performance goals in terms of the figure-of-merit parameter $I A \alpha / R^{x}$ for a number of confinement scaling expressions (see Appendix), evaluated at the density $\left(n \propto I / a^{2}\right)$ and beta $(\beta \propto I / a B)$ limits. Also listed are the scalings of $Q, P_{\text {fus }}, \Gamma_{w}$, and $P_{C D}$. The lower bound on $I A^{\alpha} / R^{\pi}$, in terms of confinement, is set by the containment of alpha particles $(\alpha=0.5, x=0)$ and by the irreducible neoclassical transport $(\alpha=3 / 8, x=0)$. All of the scalings show a weaker dependence on size $(x$ $0.1-0.5$ ). Power law scalings ${ }^{3,6-8}$ (Goldston, KayeGoldston, Kaye, T-10, etc.) exhibit a stronger dependence on aspect ratio $(\alpha-0.8-1.5)$ than the offset linear forms ${ }^{9-11}$ (Odajima-Shimomura, Rebut-Lallia, etc.), where $\alpha \leq 0.5$. A simple overall average scaling is $\langle\alpha\rangle \sim 1.1,\langle x\rangle \sim 0.4$, which can be used as a reasonable measure of confinement capability for designs with $A-2.5-4$. It should be noted that the results presented here should not be exurapolated to very high aspect ratios because the scaling of confinement with $A$ is one of the most uncertain elements of the present exper.:icental data base.
TABLE I.

Current, Size, and Aspect Ratio Scaling of

\begin{tabular}{|c|c|c|}
\hline Confinement scaling ${ }^{3-11}$ & At $n$ limit & At $\beta$ limit \\
\hline NC Neoclassical & $\left(I A^{3 / 8} / R^{0}\right)^{2}$ & $\left(I A^{3 / 8} / R^{0}\right)^{2}$ \\
\hline NA Neo-Alcator $(\mathrm{OH})$ & $\left(I A^{1.5} / R^{0.5}\right)^{2}$ & $\left(/ A^{1.25 / R^{0.25}}\right)^{4}$ \\
\hline G Goldston & $\left(/ A^{1.37} / R^{0.12}\right)^{2}$ & $\left(I A^{1.37 / R^{0.12}}\right)^{2}$ \\
\hline KG Kaye-Goldston & $\left(I A^{1.32 / R^{0.55}}\right)^{3}$ & $\left(I A^{1.3} / R^{0.5}\right)^{3.2}$ \\
\hline KA Kaye (All) & $\left(I A^{1.2 / R^{0.68}}\right)^{2.5}$ & $\left(I A^{1.2} / R^{0.63}\right)^{2.7}$ \\
\hline KB Kaye (Big) & $\left(I A^{0.8} / R^{0.56}\right)^{2.5}$ & $\left(I A^{0.82} / R^{0.52}\right)^{2}$ \\
\hline T10 T-10 & $\left(I A^{1.5} / R^{0.5}\right)^{2.53}$ & $\left(I A^{1.44} / R^{0.44}\right)^{2}$ \\
\hline OS Odajima-Shimomura & $\left(I A^{0} / R^{0}\right)$ & $\left(I A^{0.5} / R^{0}\right)^{2}$ \\
\hline RL Rebut-Lallia & $\left(I A 0.5 / R^{0.25}\right)^{2}$ & $\left(I A^{0.67} / R^{0.17}\right)^{3}$ \\
\hline AX ASDEX-H & $\left(I A / R^{0.56}\right)^{2}$ & $\left(I A / R^{0.33}\right)^{3}$ \\
\hline \multirow[t]{2}{*}{ "Simple Average" } & $\left(I A^{1.1} / R^{0.45}\right)^{2.4}$ & $\left(I A^{1.1} / R^{0.34}\right)^{2.8}$ \\
\hline & $\langle\alpha\rangle-1.1 ;\langle x\rangle$ & $-0.4 ;\langle z>-2$ \\
\hline$Q \quad$ Energy gain factor & $\left(L A / R^{0}\right)^{0}$ & $\left(I, 1 / R^{0}\right)^{1.0}$ \\
\hline $\bar{\Gamma}_{\mathbf{W}} \quad$ Wall loading & $\left(I A 1.5 / R^{1.5}\right)^{2}$ & $\left(I A^{1.25 / R^{0.75}}\right)^{4}$ \\
\hline$P_{\text {fus }}$ Fusion power & $\left(I A / R^{0.5}\right)^{2}$ & $\left(I A / R^{0.25}\right)^{4}$ \\
\hline$P_{C D}$ Current drive power & $\left(I A / R^{0.5}\right)^{2}$ & $\left(I A / R^{0.33}\right)^{3}$ \\
\hline
\end{tabular}
Confinement Capability

[Evaluated at $n$ or $\beta$ limit; fixed $<T>$ and $(q, \kappa, \delta)$ ]

$$
\operatorname{Tn} \tau_{\mathrm{E}} \sim f\left[\left(I A^{\alpha} / R^{\mathrm{x}}\right)^{z}\right]
$$

As an example, wilh $n=n_{\mathrm{mu}}$ and $\langle T\rangle=10 \mathrm{keV}$, Fig. 1 shows the normalized current $\left[I(A / 3)^{\alpha}\right]$ needed for ignition as a function of plasma size $(R$ or $a)$ for some of the confinement scalings given in Table I for fixed plasma shape $(\kappa=2, \delta=0.4)$ and safety factor $\left(q_{\Psi}=3-3.5\right)$. Because the neo-Alcator (NA) scaling represents an upper limit to confinement, in Fig. 1 we have used a combined form for $\tau_{E}$, taken as $\tau_{E}=\left[\left(\tau_{N A}\right)^{2}+\left(\tau_{\text {aux }}\right)^{2}\right]^{-1 / 2}$, where aux $=G, K G$, etc. It is also possible to consider $\tau_{E}=$ $\min \left(\tau_{\text {NA }} ; \tau_{\text {aux }}\right.$ ). Results from detailed analysis (and Fig. 1) indicate that, for a CIT-size device (with $R \sim 2 \mathrm{~m}$ ) the ignition requirement is $J(A / 3)^{\alpha} \sim 9-15 \mathrm{MA}$ for enhanced-L or $\mathrm{H}$-mode scalings, where $\tau_{\mathrm{E}}(\mathrm{H}$-mode $)=f \times \tau_{\mathrm{E}}$ (L-mode $)$ with an enhancement factor $f \geq 1.4-2$. For an ITER-size device (with $R-5-6 \mathrm{~m}), I(A / 3) \alpha \sim 15-25 \mathrm{MA}$ is required for ignition. A similar-size ITER with noninductive current drive and a wall loading of $\Gamma_{\mathrm{w}}-1 \mathrm{MW} / \mathrm{m}^{2}$ would require $I(A / 3) \sim 14-17 \mathrm{MA}$, in which $Q \sim 5-10$ operation is possible for various current drive schemes 12 (with varying bcotstrap contribution, 0-30\%) requiring $P_{C D}-75-150$ MW of absorbed power. 


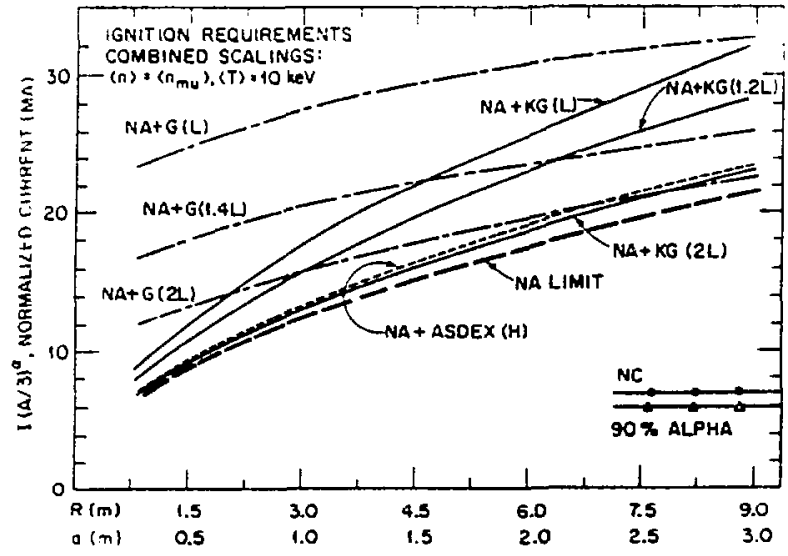

Fig. 1. Plasma current $\left[/(A / 3)^{\alpha}\right]$ required for ignition vs size $(R, a)$ for various confinement scalings (combined with neo-Alcator) for fixed plasma shape ( $\kappa=2, \delta=0.4$ ) and $q_{\Psi}=3-3.5$, evaluated at $\left(\sim n_{\text {mu }}, 10 \mathrm{keV}\right)$ (see Table I for notations).

\section{CONFINEMENT PROJECTIONS}

A detailed assessment of confinement projections for CIT and ITER has been carried out. The evaluation was based mainly on consideration of various empirical scaling expressions deduced from experiments, although theoretical models were also considered. Table II lists the representative device parameters for CIT and several ITER options and compares the confinement capabilities in terms of the figure of merit $l A^{\alpha} / R^{x}$. In Table II, the three ITER options represent the range of machines studied by the ITER design team at Garching. ${ }^{2}$ ITER-0 $(5.8 \mathrm{~m}, 20 \mathrm{MA})$ is the machine initially used to scope out physics and engineering issues. Recently, the ITER design team picked a machine with two operating phases: technology phase (ITER-1: $5.5 \mathrm{~m}, 18 \mathrm{MA}$ ) and physics phase (ITER-2: $5.8 \mathrm{~m}, 22-25 \mathrm{MA}$ ). Although there are slight differences in physics design guidelines and assumptions for CIT and ITER, for comparison purposes and uniformity we use a common set of physics assumptions $\left(Z_{\text {eff }}=1.5\right.$ with $n_{\alpha} / n_{\mathrm{e}} \approx 5 \%, \beta_{\text {crit }} \sim 3 / / a B$, etc. $)$.

As seen from Table II, all ITER versions have comparable performance, except for extended capability in the physics phase (ITER-2 with $25 \mathrm{MA}$ ) in which the performance is better. Here the performance is measured by relative magnitudes of the figure-of-merit parameter $\left(I A^{\alpha} / R^{x}\right)$. On the average, CIT and ITER confinement capabilities are also comparable; however, there are marked differences with respect to power law and offset linear forms of scalings. For the offset linear form (Rebut-Lallia, Odajima-Shimomura, etc.), performance is better in ITER (by as much as 50\%) than in CIT. With Kaye-type scalings $3,7\left(I A^{\alpha} / R^{x}\right.$ with $\left.\alpha \sim 0.8-1.3, x \sim 0.5\right)$, CIT exhibits a slightly better performance; large current in ITER is balanced with compact size and somewhat higher $A$ in CIT. With the Goldston scaling, ITER performs better due to large current and weak size scaling.
Table II.

h. presentative CIT and ITER Parameters and Confinement Capabilities

Design Parameters 1.2

CIT ITER-0 ITER-1 ITER-2

\begin{tabular}{lllll}
\hline$R$ (m) & 2.1 & 5.8 & 5.5 & 5.8 \\
$a(\mathrm{~m})$ & 0.65 & 2.0 & 1.8 & 2.2 \\
$A=R / a$ & 3.25 & 2.9 & 3.1 & 2.6 \\
$K$ (at 95\% flux) & 2 & 2 & 1.9 & 1.9 \\
$\delta$ (at 95\% flux) & 0.4 & 0.4 & 0.4 & 0.4 \\
$B$ (T) & 10 & 5.1 & 5.3 & 5.0 \\
$I$ (MA) & 11 & 20 & 18 & $22-25$
\end{tabular}

Calculated Parameters and Confinement Capability

\begin{tabular}{|c|c|c|c|c|}
\hline$q *$ (at 95\%) & 2.7 & 2.6 & 2.4 & $2.6-2.3$ \\
\hline $\begin{array}{l}q_{\psi} \text { (at } 95 \% \text { ) } \\
\text { Troyon beta limit (\%) }\end{array}$ & 3.2 & 3.2 & 2.85 & $3.2-2.9$ \\
\hline$\beta_{\text {crit }}=3 / / a B$ & 5.1 & 5.9 & 5.65 & $6-6.8$ \\
\hline $\begin{array}{l}\qquad=2.5 I / a B \\
\text { Density limit }\left(1020 \mathrm{~m}^{-3}\right)\end{array}$ & 4.2 & 4.9 & 4.7 & $5-5.7$ \\
\hline$<n_{\mathrm{mu}}>=1.5 B / R q$ & 2.6 & 0.5 & 0.6 & $0.5-0.6$ \\
\hline$\left\langle n_{\mathrm{gr}}\right\rangle=0.27 / / a^{2}$ & 7.0 & 1.35 & 1.5 & $1.2-1.4$ \\
\hline$<n\left(\right.$ at $\left.\beta_{\text {crit }}, 10 \mathrm{keV}\right)>$ & 5.9 & 1.8 & 1.85 & $1.7-2.0$ \\
\hline \multicolumn{5}{|l|}{ Figure of merit (at $\beta$ limit) } \\
\hline$<$ Average $>: / A 1.1 / R^{0.34}$ & 31 & 35.5 & 34.5 & $35-40$ \\
\hline $\mathrm{G}: \quad I A 1.37 / R 0.12$ & 50.5 & 69.5 & 68 & $67-76$ \\
\hline $\mathrm{KG}: I A^{1.3 / R^{0.5}}$ & 35 & 33 & 33 & $32-37$ \\
\hline $\mathrm{KA}: / A 1.2 / R 0.63$ & 28 & 24 & 23.5 & $23-26$ \\
\hline $\mathrm{KB}: / A^{0.82} / R^{0.52}$ & 19.5 & 19 & 18.5 & $19-22$ \\
\hline $\mathrm{RL}: I A 0.67 / R 0.17$ & 21 & 30 & 28.5 & $31-35$ \\
\hline OS: $I A^{0.5 / R^{0}}$ & 20 & 34 & 31 & $36-41$ \\
\hline
\end{tabular}

Table III summarizes the CIT and ITER ignition requirements for various scaling expressions. Given in the table are the minimum L-t node enhancement factors needed for ignition, evaluated at the beta limit (and $T-10 \mathrm{keV}$ ) assuming broad profiles and $Z_{\text {eff }}=1.5$ with $n_{\alpha} / n_{c}=5 \%$. For CIT, the predictions with Goldston and OdajimaShimomura scalings are the most pessimistic $(f \sim 2.1-2.3)$, followed by the recent Kaye ("all" and "big") scalings 3 ( $f \sim$ 1.8-1.9). Ignition with L-mode is nearly accessible with the optimistic Rebut-Lallia scaling $(f \sim 1.1)$. For ITER, the most pessimistic performance is with the recent Kaye (all and big) scaling expressions $(f-1.9-2.3)$, whereas the best performance is with the offset linear forms (Rebut-Lallia ignites with L-mode and Odajima-Shimomura requires $f$ 1.2-1.5). Projections with the T-10 scaling are uniform across the board, $f \sim 1.6$ for both CIT and the ITERs. 
Table III.

CIT and ITER Ignition Capability:

L-mode Enhancement Factor ( $f$ ) Needed for Ignition

for Various Confinement Scalings ${ }^{3-11}$

(Evaluated at Beta Limit $\beta \sim 3 / / a B \%$, fixed $T)^{a}$

\begin{tabular}{lllllll}
\hline & & CIT & \multicolumn{2}{l}{ ITER-0 ITER-1 } & ITER-2 \\
Confinement scaling & 11 MA & 20 MA & 18 MA & 22 MA \\
\cline { 1 - 2 } NA Neo-A.lcator & & Ignited & Ignited & Ignited & Ignited \\
AX ASDEX-H & Ignited & Ignited & Ignited & Ignited \\
G Goldston & $>2.1$ & $>1.6$ & $>1.6$ & $>1.6$ \\
KA Kaye ("all") & $>1.8$ & $>2.3$ & $>2.3$ & $>2.3$ \\
KB Kaye ("big") & $>1.9$ & $>2$ & $>2$ & $>1.9$ \\
T-10 T-10 & $>1.6$ & $>1.6$ & $>1.6$ & $>1.6$ \\
OS Odajima-Shimomura & $>2.4$ & $>1.3$ & $>1.5$ & $>1.2$ \\
RL Rebut-Lallia & $>1.1$ & Ignited & Ignited & Ignited
\end{tabular}

a $\tau_{\mathrm{E}}=\min \left(\tau_{\mathrm{NA}} ; \tau_{\mathrm{aux}}\right)$, aux $=\mathrm{G}, \mathrm{KA}, \mathrm{KB}, \mathrm{T}-10, \mathrm{OS}, \mathrm{RL}$

For the most part, attainment of ignition in both $\mathrm{CIT}$ and ITER relies mostly on the attainment of an enhancement ( $\mathrm{H}$-mode) over the L-mode confinement. With marginal or poor confinement (i.e., saturated ohmic and most i-mode scalings), ignition probability is very low and access to high- $Q$ operation depends sensitively on the available auxiliary power $\left(P_{\text {aux }}\right)$, which may be too large to be practical. The minimum auxiliary power requirement is obtained from the saddle point $\left(n_{*}, T_{*}\right)$ equation. For a confinement model of the form $\tau_{E} \sim n^{x} T^{y} f$ (others), where $f$ (others) contains the dependence of $\tau_{E}$ on parameters other than $n$ and $T$, the saddle point equation is 5

$$
\begin{aligned}
{[(1+\mathrm{x}) / 2] P_{\mathrm{cond}} } & =P_{\mathrm{aux}}+P_{\mathrm{OH}} \\
{[(1-\mathrm{x}) / 2] P_{\mathrm{cond}} } & =P_{\alpha}+P_{\mathrm{rad}} \\
(1-\mathrm{y}) P_{\mathrm{cond}} & =2 P_{\alpha}-1.5 P_{\mathrm{OH}}-0.5 P_{\mathrm{rad}}
\end{aligned}
$$

where $\langle\sigma v\rangle \sim T 2$ is assumed (valid for $T-7-20 \mathrm{keV}$ ). Solutions to these equations give the density and temperature at the saddle point $\left(n_{*}, T_{*}\right)$ and the required minimum $P_{\text {aux }}$ (max). The simplest, although one of the more pessimistic, of the examples is one with $\tau_{E}=$ constant (i.e., $x=y=0$ ). For reference density and temperature profiles $\left(\alpha_{n}=0.5\right.$ and $\left.\alpha_{T}=1.0\right)$,

$$
\begin{aligned}
& \min P_{\mathrm{aLx}}\left(n_{*}, T_{*}\right)=\left(s_{1} / \tau_{\mathrm{E}}-s_{2}\right) V(\mathrm{MW}) \\
& \beta\left(n_{*}, T_{*}\right)=1.68 s_{1} / \mathrm{B}^{2}
\end{aligned}
$$

where $V$ is the plasma volume, $s_{1}=5.6 \times 10^{-2}\left(1 / \mathrm{Ka}^{2}\right)$, and $s_{2}=0.85 s_{l}^{2}$.
The ignition capability can be significantly improved with centrally peaked density and heat deposition profiles. centrally peaked density profile leads to an enhanced fusion rate and therefore a greater margin against confinement losses. Although it may be possible to maintain peak profiles transiently in CIT (which operates for short pulses), attainment and sustainment of such peaked profiles over longer periods in ITER-like (long-pulse, steady-state) plasmas may not be compatible with the MHD stability and !1-mode-like conditions needed for gocd confinement.

\section{APPENDIX}

\section{Confinement Scaling Expressions}

1. Neo-Alcator (NA) OH scaling:

$\tau_{\text {NA }}=0.07 n_{20} R^{2} q$.

$q *=\left(5 a^{2} B / R I\right)\left[1+\kappa^{2}\left(1+2 \delta^{2}-1.2 \delta^{3}\right)\right] / 2$

2. Goldston (G) L-mode scaling: 6

$\tau_{\mathrm{G}}=0.037 I P-0.5 R^{1.75} a^{-0.37} \mathrm{~K}^{0.5}\left(A_{\mathrm{i}} / 1.5\right)^{0.5}$

3. Kaye-Goldston (KG) L-mode scaling:?

$\tau_{\mathrm{KG}}=C_{\mathrm{KG}}{ }^{1.24} P^{-0.58} R^{1.65} a^{-0.49} \mathrm{~K}^{0.28} n_{20^{0.26} B^{-0.09}}$

$C_{\mathrm{KG}}=0.055\left(A_{\mathrm{i}} / 1.5\right)^{0.5}$

4. Kaye "all" (KA) L-mode scaling: 3

$\tau_{\mathrm{KA}}=0.067 I^{0.85} P^{-0.5} R^{0.85} a^{0.3} \kappa^{0.25} n_{20} 0.1 B^{0.3} A_{\mathrm{i}}^{0.5}$

5. Kaye "big" (KB) L-mode scaling: 3

$\tau_{\mathrm{KB}}=0.105 \Gamma^{0.85} P^{-0.5} R^{0.5} a^{0.8} \kappa^{0.25} r_{20} 0.1 B^{0.3} A_{\mathrm{i}}^{0.5}$

\section{T-10 L-mode scaling: ${ }^{8}$}

$$
\tau_{\mathrm{T}-10}=0.095 a R B \mathrm{~K}^{0.5} \mathrm{P}-0.4\left[Z_{\mathrm{eff}}{ }^{2} I^{4} /\left(a R \varepsilon_{*}{ }^{3} \mathrm{~K}^{1.5}\right)\right]^{0.08}
$$

7. Odajima-Shimomura or JAERI (OS) L-mode scaling:9

$$
\begin{aligned}
& { }^{{ }_{\mathrm{OS}}}=C_{\mathrm{O}} \mathrm{Ka}^{2}+C_{\mathrm{S}} \ln _{20} 0.6 B^{0.2} R^{1.6} a^{0.4} \mathrm{x}^{0.2 / P} \\
& C_{\mathrm{O}}=0.085 A_{\mathrm{i}}{ }^{0.5} \\
& C_{\mathrm{S}}=0.069 G\left(q_{*}, Z_{\mathrm{cff}}\right) A_{\mathrm{i}}^{0.5} \\
& G\left(q_{*}, Z_{\mathrm{eff}}\right)=g\left(q_{*}\right) h\left(Z_{\mathrm{eff}}\right) \\
& g\left(q_{*}\right)=\left[3 q_{*}\left(q_{*}+5\right) /\left(q_{*}+2\right)\left(q_{*}+7\right)\right]^{0.6} \\
& h\left(Z_{\mathrm{eff}}\right)=Z_{\mathrm{eff}}{ }^{0.4}\left[\left(15-Z_{\mathrm{eff}}\right) / 20\right]^{0.6}
\end{aligned}
$$


- Rebut-Lallia (RL) L-mode scaling: ${ }^{10}$

$\tau_{\mathrm{RL}}=C_{\mathrm{R}} / l^{1.5}+C_{\mathrm{L}} n_{20} 0.75 P^{0.5} B^{0.5} l^{2.75} / P$

$l=\left(R a^{2} \kappa\right)^{1 / 3}$

$C_{\mathrm{R}}=\left(0.024 / Z_{\mathrm{eff}} 0.5\right)\left(A_{\mathrm{i}} / 2\right)^{0.5}$

$C_{\mathrm{L}}=0.29 Z_{\mathrm{eff}}{ }^{0.25}\left(A_{\mathrm{i}} / 2\right)^{0.5}$

9. ASDEX (AX) H-mode scaling: "1

$\tau_{\mathrm{AXH}}=0.1 / R$

In all expressions, the units are mks with currents in $\mathrm{MA}$, powers in $\mathrm{MW}$, temperatures in $\mathrm{keV}$, with $\mathrm{K}$ and $\delta$ at 95\% flux and

$$
\begin{aligned}
n_{20} & =\left\langle n_{\mathrm{e}}\right\rangle / 1020 \mathrm{~m}^{-3} \\
& =\text { volume-averaged electron density, } \\
T_{10} & =\langle T>110 \mathrm{keV} \\
& =\text { density-weighted average temperature, } \\
q_{\Psi} & \approx q \cdot f(\varepsilon)=q \cdot\left[(1.77-0.65 \varepsilon) /\left(1-\varepsilon^{2}\right)^{2}\right] ; \varepsilon=a / R \\
& =\text { MHD safety factor, } \\
A_{\mathrm{i}} \quad & =\text { average atomic mass }=2.5 \text { for a } 50: 50 \mathrm{D}-\mathrm{T} \text { plasma, } \\
Z_{\mathrm{eff}} & =\text { effective charge }=1.5 \text { (assumed for this study), } \\
P \quad & =W / \tau_{\mathrm{E}}=0.24 n_{20} T_{10}\left(1+n_{\mathrm{i}} / n_{\mathrm{e}}\right) V / \tau_{\mathrm{E}} \\
& =P_{\text {aux }}+P_{\mathrm{OH}}+P_{\mathrm{a}}-P_{\text {rad }} \\
& =\text { net "heating" power. }
\end{aligned}
$$

Ptofiles: $n, T \sim\left(1-r^{2} / a^{2}\right)^{\alpha_{n, T}}, \alpha_{n}=0.5$ and $\alpha_{\mathrm{T}}=1.0$.

\section{REFERENCES}

1. CIT papers at this meeting; see also R. PARKER et al., "CIT Physics Design Description," AE-880I12-PPl-01, Princeton Plasma Physics Laboratory (1988); D. POST et al., "Physics Aspects of the Compact Ignition Tokamak," Phys. Scr., T16, 89 (1987).

2. "TTER Definition Phase Report," International Atomic Energy Agency, Vienna (to be published); ITER Physics Team (presented by D. POST), "The Physics Basis of ITER," to appear in Proc. I2th Int. Conf. Plasma Physics and Controlled Nuclear Fusion Research, Nice, France, October 12-19, 1988.

3. For review of empirical scalings, see S. M. KA.YE, "Survey of Energy Confinement Scaling Expressions," ITER Specialists' Meeting on Energy Confinement, May 24-27, 1988, Garching, Federal Republic of Germany.

4. For review of theoretical models, see J. D. CALLEN, "Overview of Confinement Theory," ITER Specialists" Meeting on Energy Confinement, May 24-27, 1988, Garching, Federal Republic of Germany.
5. N. A. UCKAN and J. SHEFFIELD. "A Simple Procedure for Establishing Ignition Conditions in Tokamaks," Tokamak Startup, p. 45, H. Knoepfel, Ed., Plenum Press, New York (1986); N. A. UCKAN, "Relative Merits of Size, Field, and Current on Ignited Tokamak Performance," Fusion Technol., 14, 299 (1988).

6. R. J. GOLDSTON, "Energy Confinement Scaling in Tokamaks," Plasma Phys. Controlled Fusion. 26, 87 (1984).

7. S. M. KAYE and R. J. GOLDSTON, "Global Energy Confinement Scaling of Neutral Beam Heated Tokamaks," Nucl. Fusion, 25, 65 (1985).

8. T-10 GROUP, "Investigation of Energy Confinement in ECH Experiments on T-10," ITER Specialists' Meeting on Energy Confinement, May 24-27, 1988, Garching, Federal Republic of Germany.

9. K. ODAJIMA and Y. SHIMOMURA, "Energy Confinement Scaling Based on Offset Linear Characteristics," JAERI Repor, JAERI-M 88-068 (March 1988).

10. P. H. REBUT, P. P. LALLLA, and M. L. WATKINS, "Chaotic Magnetic Topology and Heat Transport in Tokamaks," JET Report, JET-P(88)05 (January 1988, corrigendum March 1988).

11. O. GRUBER, "Confinement Regimes in Ohmically and Auxiliary Heated Tokamaks," Proc. Int. Canf. Plasma Physics, Lausanne, Switzerland, June 27-july 3, 1984, Vol. 1, p. 67, Commission of the European Communities (1984).

12. For an overview, see N. J. FISCH, "Theory of Current Drive in Plasmas," Rev. Mod. Phys., 59, 175 (1987). 